\title{
EXTENSIONS OF THE JORDAN-BROUWER SEPARATION THEOREM AND ITS CONVERSE
}

\author{
PAUL A. WHITE
}

In Wilder's colloquium [2] a Jordan-Brouwer type separation theorem is proved for generalized manifolds (see Definition 2). It states that if $K$ is a connected orientable $(n-1)-\mathrm{gcm}$ (generalized closed manifold) in a connected orientable $n$-gcm $S$ such that $p^{n-1}(S)=0$ (the $(n-1)$-Betti number), then $S-K$ is the union of disjoint domains $A$ and $B$ that have $K$ as their common boundary. It is shown in this paper that under the conditions of the above theorem, the sets $\bar{A}=A \cup K$ and $\bar{B}=B \cup K$ are each generalized manifolds with boundary $K$ (see Definition 2). Furthermore if we are interested in proving only that $\bar{A}$ and $\bar{B}$ are manifolds with boundary, this can be done without the hypothesis $p^{n-1}(S)=0$ and without assuming $K$ is connected or orientable. The result then becomes the following: If $S-K=A \cup B$ separate, where $K$ is an $(n-1)$-gcm in the connected orientable $n$-gcm $S$, then $\bar{A}$ and $\bar{B}$ are generalized manifolds with boundaries consisting of some of the components of $K$. More generally it can be said that the closure of each component of $S-K$ is a gm with boundary formed by some of the components of $K$ (if $S-K$ is disconnected).

Wilder also considers converses of the Jordan-Brouwer theorem and other related theorems which are all concerned with the case where the boundary of a ulcr (uniformly locally-s-connected $s \leqq r$ ) open subset of a connected orientable $n$-gcm is a connected orientable $(n-1)-\mathrm{gcm}$. We answer the more general question as to when the closure of such a set is a gm with boundary. As before the hypothesis $p^{n-1}(S)=0$ can be eliminated if connectedness is not required in the conclusion. Thus we show among other things that the closure of an open ulc $\mathrm{c}^{n-1}$ subset of a connected orientable $n$-gcm whose boundary is ( $n-1)$-dimensional is an $n$-gm with boundary. In order to eliminate the assumption $p^{n-1}(S)=0$ it is necessary to prove an extension of the Alexander type duality theorem which does not include that hypothesis. The extended result states that if $K$ is a closed subset of the connected orientable $n$-gcm $S$ such that $S-K$ has $m$ components ( $m$ may be infinite), then $m-1 \leqq p^{n-1}(K) \leqq(m-1)+p^{n-1}(S)$.

Throughout the paper we shall assume that the space $S$ is a compact Hausdorff space. The homology theory used will be that of

Presented to the Society, April 23, 1951; received by the editors December 9, 1950. 
Cech in which the coefficient group for the chains will be an arbitrary field which we shall omit from the notation for a chain. We shall use " $\cup$ " for point set union or sum, and " $\cap$ " for intersection, reserving + and - for the group operations.

Definition 1. If $K$ is a closed subset of the closed subset $M$ of $S$, then we shall say that the local $r$-dimensional Betti number of $M$ at $x$ $\bmod K\left(\right.$ denoted by $\left.p_{r}(M \bmod K, x)\right)$ is the finite integer $k$ if $k$ is the smallest positive integer with the property that corresponding to any open set $P$ such that $x \in P$ there exists an open set $Q$ such that $x \in Q \subset \subset P$ (i.e., $\bar{Q} \subset P$ ) and such that any $k+1$ Cech cycles of $M \bmod (M-(P-K))=(M-P) \cup K$ are linearly dependent with respect to homologies on $M \bmod M-(Q-K)=(M-Q) \cup K$. (Note that if $K=0$ then this definition is equivalent to the definition of $p_{r}(M, x)$, the local Betti number of $M$ at $x$. Also this is equivalent to Wilder's definition on p. 291 of [2] of the Betti number around a point.)

Definition 2. The compact space $M$ will be called an $n$-dimensional generalized manifold ( $n$-gm) with boundary if there exists a closed subset $K$ of $M$ such that

(a) $M=K \cup A$ where $A$ is open and $K=\bar{A}-A$,

(b) $\operatorname{dim} M=n$ (in the sense of Lebesgue, see p. 195 of [2]),

(c) $p_{r}(M \bmod K, x)=0$ for all $x \in M, r \leqq n-1$,

(d) $p_{n}(M \bmod K, x)=1$ for all $x \in M$,

(e) $p_{r}(M, x)=0$ for all $x \in K, r \leqq n$.

(Note that this definition reduces to Wilder's definition of an $n$ dimensional generalized closed manifold $(n-\mathrm{gcm})$ when $K=0$ (see p. 244 of [2]).)

Definition 3. An $n$-gm $M$ with boundary $K$ is called orientable if $M$ is the carrier of a cycle $z^{n} \bmod K$ such that ${ }^{1} z^{n} \sim_{n} \Gamma^{n} \bmod K$ on $M$ where $\Gamma^{n}$ is a cycle $\bmod K$ on a proper closed subset of $M$. (If $K=0$, this becomes the definition of an orientable $n$-gcm.)

LEMma 1. If $S$ is a connected orientable $n$-gcm and $K$ is a closed subset of $S$ such that $S-K=A \cup B$ separate, and $K$ is the common boundary of $A$ and $B$, then $p_{n}(A \cup K, x)=p_{n}(B \cup K, x)=0$ for every $x \in K$.

PRoof. Suppose $x \in K$ and $x \in U$, an open subset of $S$. Let $M$ $=A \cup K$ and $N=B \cup K$. Since $S$ is $n$-dimensional, there is a cofinal family of $n$-dimensional coverings of $S$, and we shall suppose that all Cech cycles and chains have coordinates only on these coverings. Let $z^{n}$ be such a Cech cycle on $M \bmod M-U C S-U$, and suppose that no open set $V$ exists, $x \in V \subset \subset U$ such that $z^{n} \sim 0 \bmod S-V$. This

$1 \sim_{n}$ means not $\sim$. 
implies that there is one such $V$ and an element $f \neq 0$ of the coefficient group, such that $z^{n} \sim f \gamma^{n} \bmod S-V$ where $\gamma^{n}$ is the fundamental nonbounding $n$-cycle of $S$. Choose $y \in V \cap B$ and let $W$ be an open set such that $y \in W \subset \subset V \cap B$; hence $W \cap M=0$. Now $z^{n}$ is on $S-W$; therefore $\gamma^{n} \sim(1 / f) z^{n} \sim 0$ mod $S-W$ which is impossible by Lemma 3.6 on p. 214 of [2]. Thus $z^{n} \sim 0 \bmod S-V$ for some $V$, hence $=0$ on $V$ since only $n$-dimensional coverings are considered, hence $\sim 0 \bmod M-V$, and $p_{n}(M, x)=0$.

Lemma 2. Under the hypotheses of Lemma $1, K$ carries an ( $n-1)$ cycle $\gamma^{n-1}$ that is not $\sim$ to a cycle on any proper closed subset of $K$, and $\gamma^{n-1} \sim 0$ on $A \cup K$ and $B \cup K$.

Proof. As in the proof of Lemma 1, let $M=A \cup K$ and $N=B \cup K$, and suppose all Cech cycles to have coordinates only on an $n$-dimensional cofinal family of coverings. Let $\gamma^{n}$ be the fundamental nonbounding cycle of $S$, then $\gamma^{n}$ is a cycle mod $S-A$; hence by Lemma 1.16 on p. 204 of [2] there exists a cycle $z^{n}$ on $M \bmod K$ such that $z^{n} \sim \gamma^{n} \bmod S-A$ on $S$. By Lemma 1.1 on p. 200 of [2], $\gamma^{n-1}$ $=\left\{\partial z^{n}(U)\right\}$ is an $(n-1)$-cycle on $K$. Suppose $\gamma^{n-1} \sim \gamma_{1}^{n-1}$ where $\gamma_{1}^{n-1}$ is a cycle on a proper closed subset $K_{1}$ of $K$. Thus $\partial z^{n} \sim 0 \bmod$ $K_{1}$, and by Lemma 1.7 on p. 202 of [2], there exists a cycle $z_{1}^{n} \bmod K_{1}$ on $M$ such that $z^{n} \sim z_{1}^{n} \bmod K$ on $M$. Choose $y \in K-K_{1}$ and $U$ an open set such that $y \in U, U \cap K_{1}=0$. Since, by Lemma $1, p_{n}(M, y)$ $=0$, there exists an open set $V, y \in V \subset \subset U$, such that every cycle on $M \bmod M-U$ is $\sim 0 \bmod M-V$. In particular $z_{1}^{n}$ is a cycle mod $K_{1} \subset M-U$; hence $\sim 0 \bmod M-V$. Choose $z \in(V-K) \cap A$ and an open set $W, z \in W \subset \subset V \cap A$; hence $W \cap K=0$. Since only $n$-dimensional coverings are considered, we conclude that $\gamma^{n}=z^{n}$ in $A, z^{n}=z_{1}^{n}$ in $A, z_{1}^{n}=0$ in $V$; hence $\gamma^{n}=0$ on $W$. This implies $\gamma^{n} \sim 0 \bmod S-W$ (with respect to all coverings) which contradicts Lemma 3.6 on p. 214 of (2). This shows that $\gamma^{n-1}$ is not $\sim$ to any cycle on a proper closed subset of $K$. Furthermore the method of proof shows that $\gamma^{n-1} \sim 0$ on $M$ (and similarly on $N$ ).

THEOREM 1. Let $S$ be a perfectly normal, connected, orientable $n-\mathrm{gcm}$ and $K$ an (n-1)-gcm $\subset S$, such that $S-K=A \cup B$ separate, and such that $K$ is the common boundary of $A$ and $B$; then $K$ is orientable and $M=K \cup A$ and $N=K \cup B$ are each orientable n-gm's with boundary $K$.

Proof. The orientability of $K$ follows from Lemma 2 .

It follows from Lemma 1 that $p_{r}(M, x)=0$ for all $x \in K$, when $r=n$. We shall now prove that the result holds for $r=0, \cdots, n-2$. To this end we note that the proof of the remark on p. 295 of [2] shows 
that $A$ and $B$ are each $r$-ulc, $r=0,1, \cdots, n-1$. By Theorem 2.12 on p. 294 of [2], this implies that $A$ and $B$ are $r$-coulc, $r=1, \cdots$, $n-1$. By Corollary 2.10 on p. 294 of [2], we have $\operatorname{pr}(S-B, x)$ $=p_{r}(M, x)=0, r=0, \cdots, n-2$ for all $x \in M$.

Finally we show that $p_{n-1}(M, x)=0$ for all $x \in K$. By Theorem 10 of (1) each of the finite number of components of $K$ is a connected orientable $(n-1)$-gcm. Consider $x \in K$ and let $K^{\prime}$ be the component of $K \supset x$. Choose an open set $U, x \in U$, such that $U \cap\left(K-K^{\prime}\right)=0$, and a cycle $z^{n-1} \bmod M-U$ on $M$. Since $A$ is $(n-1)$-coulc, we have by Theorem 2.8 on p. 293 of [2] that $p_{n-1}(M \bmod K, x)=0$. It follows that we can find a $V, x \in V \subset \subset U$, such that $z^{n-1} \sim 0 \bmod (M-V)$ $\cup K$ on $M$. By Lemma 1.9 on p. 202 of [2] there is a cycle $z_{1}^{n-1} \bmod M$ $-U$ on $(M-V) \cup K$ such that $z^{n-1} \sim z_{1}^{n-1} \bmod M-U$. By Lemma 1.16 on p. 204 of [2] there exists a cycle $z_{2}^{n-1} \bmod F(V)$ (the boundary of $V)$ on $[(M-V) \cup K]-(M-V)=V \cap K$ such that $z_{1}^{n-1} \sim z_{2}^{n-1} \bmod M$ $-V$ on $(M-V) \cup K$. Since $p_{n-1}(K, x)=1$, an open set $W$ exists such that $x \in W \subset \subset V$ and such that only one cycle mod $K-V$ exists that is linearly independent with respect to homologies $\bmod K-W$ (the set $V$ used above may be chosen since any open set inside the original $V$ would have done as well in that discussion). By Lemma 3.6 p. 214 of [2], the fundamental nonbounding $(n-1)$-cycle, $\gamma_{1}^{n-1}$, of $K^{\prime}$ is not $\sim 0 \bmod K-W$; therefore $z_{2}^{n-1} \sim f \gamma_{1}^{n-1} \bmod K-W$ where $f$ is an element of the coefficient field (possibly 0 ). Of course $\gamma_{1}^{n-1} \sim \gamma^{n-1}$ $\bmod K-W$ where $\gamma^{n-1}$ is the fundamental nonbounding cycle of $K$ since $\gamma_{1}^{n-1}$ is the part of $\gamma^{n-1}$ on $K_{1}$ and $W \cap\left(K-K^{\prime}\right)=0$. By Lemma 2 we have $\gamma^{n-1} \sim 0$ on $M$, hence $\sim 0 \bmod M-W$, but $z_{2}^{n-1} \sim f \gamma_{1}^{n-1}$ $\sim f \gamma^{n-1} \sim 0 \bmod M-W$. Also $z^{n-1} \sim z_{1}^{n-1} \sim z_{2}^{n-1} \bmod M-W$; therefore $z^{n-1} \sim 0 \bmod M-W$ and $p_{n-1}(M, x)=0$. Combining previous results with this gives $\operatorname{pr}_{r}(M, x)=0$ for all $x \in K$ and all $r=0,1, \cdots, n$.

Since $A$ is an open subset of an $n$-gcm, it is a noncompact $n$-gm; hence, by Theorem 2 of [1], $M$ is an $n$-gm with boundary $K$. It remains to show that $M$ is orientable. To this end consider the fundamental nonbounding $n$-cycle $\gamma^{n}$ of $S$. It was shown in Lemma 2 that a cycle $z^{n} \bmod K$ on $M$ exists such that $z^{n} \sim \gamma^{n} \bmod N$ on $S$. Suppose $z^{n}$ were $\sim \bmod K$ to $z_{1}^{n}$, a cycle $\bmod K$ on a proper closed subset $M_{1}$ of $M$. Since $\bar{A}=M, A \nsubseteq M_{1}$, and $M_{1} \cup N=S_{1}$ is a proper closed subset of $S$. Let $U=$ the nonempty open set $S-S_{1}$. Since $N$, $K, M_{1}$ are all $\subset S-U$, we have $\gamma^{n} \sim z^{n} \sim z_{1}^{n} \sim 0 \bmod S-U$ contrary to Lemma 3.6 on p. 214 of (2). Thus $z^{n}$ is not $\sim \bmod K$ to a cycle on any proper closed subset of $M$; therefore $M$ is orientable. Of course the same argument shows that $N$ is also an orientable $n$-gm with boundary $K$. 
COROLLARY 1.1. If $S$ is a connected orientable $n-g c m$, such that $p^{n-1}(S)$ $=0$, and $K \subset S$ is a connected $(n-1)-g c m$, then $S-K=A \cup B$ separate and $A \cup K, B \cup K$ are each orientable $n$-gms with common boundary $K$.

Proof. Theorem 3.1 on p. 294 of [2] (the generalized JordanBrouwer separation theorem) tells us that $S-K=A \cup B$ separate such that $K$ is the common boundary of $A$ and $B$. The remainder of the corollary now follows from the theorem.

Of course the hypothesis $p^{n-1}(S)=0$ is necessary to insure the separation of $S-K$ as is seen by considering the torus, but one may ask whether the fact that $\bar{A}$ and $\bar{B}$ are generalized manifolds with boundary is dependent on that fact. The following two theorems and their corollaries give a negative answer to that question.

THEOREM 2. If $S$ is a connected orientable n-gcm, and $K \subset S$ is an $(n-1)-g c m$, then each component of $K$ is either part of the boundary of exactly one or exactly two components of $S-K$.

If $S-K$ is connected, the conclusion follows immediately, so we shall assume in what follows that $S-K$ is disconnected. Let $S-K$ $=\bigcup A_{i}$ where $\left\{A_{i}\right\}$ are the components of $S-K$ and are all open since $S$ is lc. Clearly $F\left(A_{i}\right) \subset K$ for each $i$. Consider $x \in K$. Since $p_{n}-1(K, x)=1$, we conclude by Theorem 1.5 on p. 292 of [2] that there exist open sets $P, Q, x \in Q \subset P$, such that exactly one (augmented) compact 0 -cycle of $Q-K$ is linearly independent with respect to homology in $P-K$. By Theorems 11.10 on p. 143 and 3.3 on p. 105 of [2], this implies that $Q-K$ lies in exactly 2 components of $P-K$. Since $\left\{(P-K) \cap A_{i}\right\}$ are multiwise separated, this implies that at most 2 of the sets $A_{i}$ meet $Q$. Suppose $A_{1}$ and $A_{2}$ meet $Q$; hence $Q-K=\left(A_{1} \cap Q\right) \cup\left(A_{2} \cap Q\right)$ and since any $R \subset Q$ has the same properties as $Q, x$ is a limit point of $A_{1}$ and $A_{2}$. Let $K_{1}$ be the component of $K \supset x$ and suppose there exists a $y \in K_{1}$ which is not a limit point of both $A_{1}$ and $A_{2}$. Since $K_{1}$ is a locally connected continuum, there exists an arc from $x$ to $y$ and a last point $z$ in that direction on the arc that is a limit point of $A_{1}$ and of $A_{2}$. Let $z \in Q^{\prime} \subset P^{\prime}$ where $Q^{\prime}$ and $P^{\prime}$ are the open sets corresponding to $z$ described above, i.e., $R^{\prime}-K \subset A_{1} \cup A_{2}$ where $R^{\prime}$ is any open set $\supset z, \subset Q^{\prime}$. Choose $t \in Q^{\prime}$ and on the subarc from $z$ to $y$. Clearly all open sets $S^{\prime} \supset t, S^{\prime} \subset Q^{\prime}$ have the property that $S^{\prime}-K=\left(A_{1} \cap S^{\prime}\right) \cup\left(A_{2} \cap S^{\prime}\right)$ and $t$ is a limit point of $A_{1}$ and of $A_{2}$ contrary to the assumption that $z$ was the last such point. Thus every point of $K_{1}$ is a limit point of $A_{1}$ and of $A_{2}$. It also follows from this that if $x$ is a limit point of just $A_{1}$, for example, then all points of $K_{1}$ are limit points of just $A_{1}$. These two facts imply the conclusion. 
THEOREM 3. If $S$ is a perfectly normal, connected orientable $n-g \mathrm{~cm}$, and $K \subset S$ is an $(n-1)-g c m$ such that $S-K$ is disconnected, then the closure of each component of $S-K$ is an orientable $n$-gm with a boundary consisting of some of the components of $K$.

Proof. Let $S-K=\cup A_{i}$ where $\left\{A_{i}\right\}$ are the components of $S-K$. As stated in the proof of Theorem 2, these components are all open and, therefore, multiwise separate. Let $K^{\prime}=\bar{A}_{1} \cap$ closure of $\bigcup A_{i}$ $(i \neq 1) . K^{\prime} \subset K$ since closure of $\bigcup A_{i}(i \neq 1) \cap A_{1}=0=\bar{A}_{1} \cap \bigcup A_{i}(i \neq 1)$; in particular each point of $K^{\prime}$ is a limit point of $A_{1}$. Furthermore by the proof of the preceding theorem, there exists for each $x \in K^{\prime}$ an open set $Q$ such that $Q-K=\left(A_{1} \cap Q\right) \cup\left(A_{j} \cap Q\right), 1 \neq j$, or $Q-K$ $=A_{1} \cap Q$. The latter case is impossible since each point of $K^{\prime}$ is a limit point of $U A_{i}(i \neq 1) \subset S-A_{1}$; therefore, by Theorem 2 each point of $K^{\prime}$ is on the boundary of exactly two components of $S-K$, one of which is $A_{1}$. Now suppose $K^{\prime} \cap K_{1} \neq 0$ where $K_{1}$ is a component of $K$; hence by Theorem 2 all of $K_{1}$ is on the boundary of exactly $2 A_{i}$ 's, $A_{1}$ and some $A_{j}, j \neq 1$. Thus $K_{1} \subset \bar{A}_{1} \cap \bar{A}_{j} \subset K^{\prime}$, and we see that $K^{\prime}$ consists of several of the components of $K$ and is, therefore, itself an $n$-gcm. Since $S-K^{\prime}=\left(\bar{A}_{1}-K^{\prime}\right) \cup$ (closure of $\left.\cup A_{i}(i \neq 1)-K^{\prime}\right)$ separate, we conclude by Theorem 1 that $\bar{A}_{1}$ is an $n$-gm with boundary $K^{\prime}$.

CoROLlaRY 3.1. If $S$ is a perfectly normal connected orientable n-gcm and $K \subset S$ is an $(n-1)-g c m$, then $S-K$ has only a finite number of components.

Proof. By the proof of Theorem 3 each of the components of $K$ is on the boundary of at most 2 components and the boundary of each component lies in $K$. Since the number of components of $K$ is finite, it follows that the same holds for $S-K$.

CoRollaRY 3.2. If $S$ is a perfectly normal connected orientable $n$-gcm and $K \subset S$ is an (n-1)-gcm, such that $S-K=A \cup B$ separate, then $\bar{A}$ and $\bar{B}$ are orientable $n$-gms with boundaries $K_{A}$ and $K_{B}$ each of which is a union of components of $K$.

Proof. The proof of Theorem 3 can be used to show that $\bar{A} \cap \bar{B}$ is an $(n-1)$-submanifold of $K$. Now the result follows from Theorem 1 with $\bar{A} \cap \bar{B}$ taking the place of $K$ in the statement of that theorem.

On p. 296 of [2], Wilder has stated the following converse of the Jordan-Brouwer separation theorem: "If $K$ is an $(n-1)$-dimensional closed subset of a connected orientable $n$-gcm $S$ such that $p^{n-1}(S)=0$ and $S-K$ is the union of exactly $2 r$-ulc $(r=0,1, \cdots, n-2)$ domains of which $K$ is the common boundary, then $K$ is a connected 
orientable $(n-1)-\mathrm{gcm}$. In the preceding theorems, we saw that the hypothesis $p^{n-1}(S)=0$ was essentially unnecessary and we naturally ask again the question as to whether that hypothesis can be eliminated. It turns out that $p^{n-1}(S)=0$ can be omitted, but that $K$ need not be connected in that case. It is also not necessary to assume that $A$ and $B$ are domains. This result is embodied in Theorem 5 . Before proving this theorem we first prove a generalization of part of the Alexander type duality theorem, which does not use the hypothesis $p^{n-1}(S)=0$. Without this hypothesis, we can of course obtain only inequalities.

THEOREM 4. If $S$ is a connected orientable $n$-gcm and $K$ is a closed subset of $S$, such that $S-K$ has $m$ components ( $m$ can equal $\infty$ ), then $m-1 \leqq p^{n-1}(K) \leqq(m-1)+p^{n-1}(S)$. (If $p^{n-1}(S)=0$, this reduces to the ordinary Alexander type duality theorem between the $(n-1)$-dimensional Betti number of $K$ and the 0-dimensional Betti number of $S-K$.)

Proof. The first inequality $m-1 \leqq p^{n-1}(K)$ is the conclusion of Corollary 3.3 on p. 213 of [2]. In particular if $m=\infty$, then $p^{n-1}(K)$ $=(m-1)+p^{n-1}(S)=\infty$; therefore, we shall consider only the case where $m$ is finite. Let $C_{i}, i=1, \cdots, m$, be the component of $S-K$. Each $C_{i}$ is open since $S$ is 0 -lc; therefore, the boundary $F\left(C_{i}\right)$ of each $C_{i}$ is $\subset K$.

We now show that each $\bar{C}_{i}$ satisfies property $\mathrm{D}$ with respect to $F\left(C_{i}\right)$; i.e. every $n$-cycle $\bmod F\left(C_{i}\right)$ on a proper closed subset of $\bar{C}_{i}$ is $\sim 0 \bmod F\left(C_{i}\right)$. To this end let $z^{n}$ be a cycle $\bmod F\left(C_{i}\right)$ on the proper closed subset $C^{\prime}$ of $\bar{C}_{i}$. By Lemmas 2.3 and 2.6 on pp. 205-206 of [2], there exists a minimal closed set $F, C^{\prime} \supset F \supset F\left(C_{i}\right)$, such that $z^{n} \sim 0 \bmod F$ and $F$ is a carrier of $z^{n}$. Suppose $F \subset F\left(C_{i}\right)$; then there exists a point $x \in C_{i}$ on the boundary of $F$ with respect to $\bar{C}_{i}$, since $C_{i}$ is connected. Let $P$ be an open set $\supset x$ such that $P \subset C_{i}$; then there exists an open set $Q$ such that $x \in Q \subset P$ and such that there is only one $n$-cycle mod $S-P$ linearly independent with respect to homologies mod $S-Q$. The fundamental nonbounding cycle $\gamma^{n}$ of $S$ is of course such a cycle. However, $z^{n}$ cannot be linearly dependent on $\gamma^{n}$ $\bmod S-Q$ since for any open set $R \subset Q \cap(S-F)$, we have $z^{n} \sim 0 \bmod S$ $-R$, but $\gamma^{n}$ cannot have this property by Lemma 3.6 on p. 214 of [2]. We, therefore, conclude that $z^{n} \sim 0 \bmod S-Q$, which implies $z^{n}$ lies on $(S-Q) \cap F$ (if only an $n$-dimensional cofinal family of coverings of $S$ is considered). This contradicts the fact that $F$ was minimal with respect to the property $z^{n} \sim 0 \bmod F$; therefore, $F \subset F\left(C_{i}\right)$ and $z^{n} \sim 0 \bmod F\left(C_{i}\right)$.

It also follows from the proof of Theorem 5 of [1] that for any set $M$ 
with property $\mathrm{D}$ relative to $K, p^{n}(M, K) \leqq 1$; thus $p^{n}\left(C_{i}, F\left(C_{i}\right)\right) \leqq 1$. Now if $\gamma^{n}$ is the fundamental nonbounding cycle of $S$, then by Lemma 1.15 on p. 204 of [2], we have $\gamma^{n} \sim \sum_{i=1}^{m} \gamma_{i}^{n} \bmod K$ on $S$ where $\gamma_{i}^{n}$ is a cycle on $\bar{C}_{i} \bmod F\left(C_{i}\right)$ for each $i$. Finally $\gamma_{i}^{n}$ is not $\sim 0 \bmod F\left(C_{i}\right)$ on $\bar{C}_{i}$ for any $i$; for suppose the contrary for $i=1$, then $\gamma^{n} \sim \sum_{i=2}^{m} \gamma_{i}^{n}$ (or 0 if $m=1$ ) $\bmod K$ and hence $\gamma^{n} \sim 0 \bmod S-C_{1}$ contrary to Lemma 3.6 on p. 214 of [2]. This shows that $p^{n}\left(\bar{C}_{i}, F\left(C_{i}\right)\right)=1$ for each $i$.

We shall use the above facts to show that any $z^{n-1}$ on $K \sim 0$ on $M$ is linearly dependent on the cycles $\gamma_{i}^{n-1}=\partial \gamma_{i}^{n}$ on $K$. Consider such a $z^{n-1}$; then there exists a cycle $z^{n}$ on $S \bmod K$ such that $\partial z^{n} \sim z^{n-1}$ on $K$. By Lemma 1.15 on p. 204 of [2], $z^{n} \sim \sum_{i=1}^{m} z_{i}^{n} \bmod K$ on $S$ where $z_{i}^{n}$ is a cycle on $\bar{C}_{i} \bmod F\left(C_{i}\right)$ for each $i$. By the above we have $z_{i}^{n} \sim f_{i} \gamma_{i}^{n} \bmod F\left(C_{i}\right) \subset K$ where for each $i, f_{i}$ is an element of the coefficient group. By Lemma 1.2 on p. 200 of [2], this implies that $\partial z_{i}^{n}$ $\sim f_{i} \partial \gamma_{i}^{n}=f_{i} \gamma_{i}^{n-1}$; therefore by a second application of this lemma $z^{n-1} \sim \partial z^{n} \sim \sum_{i=1}^{n} \partial z_{i}^{n} \sim \sum_{i=1}^{m} f_{i} \gamma_{i}^{n-1}$ on $K$.

Actually $z^{n-1}$ is linearly dependent on the cycles $\gamma_{i}^{n-1}, i=2, \cdots, m$. For $\partial\left(\sum_{i=1}^{m} \gamma_{i}^{n}\right)=\sum_{i=1}^{m} \gamma_{i}^{n-1}$ and $\gamma^{n} \sim \sum_{i=1}^{m} \gamma_{i}^{n} \bmod K$; hence $0=\partial \gamma^{n}$ $\sim \sum_{i=1}^{m} \gamma_{i}^{n-1}$ on $K$ by Lemma 1.2 on p. 200 of [2] or $\gamma_{1}^{n-1}$ $\sim-\sum_{t=2}^{m} \gamma_{i}^{n-1}$ on $K$.

The cycles $\gamma_{i}^{n-1}, i=2, \cdots, m$, on $K$ may form a generating system for all cycles of $K$; if not there exists a cycle $\gamma_{m+1}^{n-1}$ that cannot be expressed as a linear combination of the $\gamma_{i}^{n-1}, i=2, \cdots, m$. Again the $\gamma_{i}^{n-1}, i=2, \cdots, m+1$, might form a generating system or there might be a cycle $\gamma_{m+2}^{n-1}$ independent of these, etc. Since $S$ is lc $^{n}$, we know that $p^{n-1}(S)$ has some finite value $s$. From this we can conclude that the above sequence of alternatives must result in the first alternative in a finite number of steps. For suppose in the above argument we arrive at a $\gamma_{i}^{n-1}, i=2, \cdots, m+s$, that do not form a generating system for cycles of $K$; then there exists a cycle $\gamma_{m+s+1}^{n-1}$ on $K$ independent of the other $\gamma_{i}^{n-1}$ on $K$. However the cycles $\gamma_{m+1}^{n-1}, \cdots$, $\gamma_{m+s+1}^{n-1}$ are linearly dependent in $S$ since $p^{n-1}(S)=s$; therefore there exists a linear combination $\sum_{i=m+1}^{m+s+1} f_{i} \gamma_{i}^{n-1} \sim 0$ on $S$ where not all the $f_{i}=0$. By the preceding argument this implies the existence of a homology $\sum_{i=m+1}^{m+s+1} f_{i} \gamma_{i}^{n-1} \sim \sum_{i=2}^{m} f_{i} \gamma_{i}^{n-1}$ on $K$, but the assumption that no $\gamma_{i}^{n-1}(i=m+1, \cdots, m+s+1)$ is dependent on the preceding $\gamma_{i}^{n-1}$ yields $f_{m+s+1}=\cdots=f_{m+1}=0$, which is a contradiction. Thus $p^{n-1}(K) \leqq(m-1)+s$.

We obtain immediately the following corollary.

CoROLlaRY 4.1. If $S$ is a connected orientable $n$-gcm and $K$ a closed subset, such that $p^{n-1}(K)>p^{n-1}(S)$, then $K$ separates $S$. 
An immediate consequence of this corollary and previous theorems is the following natural generalization of the Jordan-Brouwer separation theorem.

THEOREM 5. If $S$ is a perfectly normal connected orientable $n$-gcm and $K \subset S$ is an $(n-1)-g c m$ such that $p^{n-1}(K)>p^{n-1}(S)$, then $K$ separates $S$ into a finite number of components and if $S-K$ is expressed as $A \cup B$ separate, then $\bar{A}$ and $\bar{B}$ are each orientable $n$-gms with a common boundary that is an (n-1)-sub-gcm of $K$.

THEOREM 6. If $K$ is a (n-1)-dim subset of the perfectly normal connected orientable $n$-gcm $S$ such that $S-K=A \cup B$ separate, $A$ and $B$ are each $r$-ulc $(r=0,1, \cdots, n-2)$, and $K$ is the common boundary of $A$ and of $B$, then $K$ is an orientable $(n-1)-g \mathrm{~cm}$.

Proof. By Lemma $2, K$ carries an $(n-1)$-cycle $\gamma^{n-1}$ that is not $\sim$ to a cycle on any proper closed subset of $K$. Thus if $K$ is an $(n-1)-\mathrm{gcm}$ it is orientable.

We shall show that $A$ and $B$ each have only a finite number of components. To this end we note by Corollary 2.6 on p. 293 of [2] that each point $x \in S-A$ has the property that if $P$ is an open set with $x \in P$, there exists an open set $Q, x \in Q \subset P$, such that every compact 0-cycle on $Q \cap A$ is $\sim 0$ in $P \cap A$. By 11.10 on p. 143 of [2], this implies that only one component of $P \cap A$ meets $Q \cap A$. Now choose one point in each component of $A$; if their number is infinite, a subsequence will converge to a point $x \in K \subset S-A$. These components are all open and disjoint and infinitely many intersect $Q$. Since no two intersecting $Q$ can lie in the same component of $P$, we have a contradiction, and we conclude that $A$, and similarly $B$, and hence also $A \cup B=S-K$, each have only a finite number of components.

We conclude from Theorem 4 that $p^{n-1}(K)$ is finite. We shall show that the number of components of $K$ is also finite, for if not then $K$ can be written as $K_{1} \cup K_{2} \cup \ldots \cup K_{m+s}$ where the $K_{i}$ are disjoint closed sets, $m=$ the number of components of $S-K$, and $s=p^{n-1}(K)$. Now consider the cycle $\gamma^{n-1}$ described in the first paragraph of the proof. By Lemma 1.15 on p. 204 of [2], $\gamma^{n} \sim \sum_{i=1}^{m+s} \gamma_{i}^{n-1}$ on $K$ where $\gamma_{i}^{n-1}$ is a cycle on $K_{i}$ for each $i$. No $\gamma_{i}^{n-1}$ is $\sim 0$ on $K$, for $\gamma^{n-1}$ would then be $\sim$ to a cycle on a proper closed subset of $K$. Since the sets $K_{i}$ are closed and disjoint, this implies that the $\gamma_{i}^{n-1}$ are linearly independent on $K$, contrary to $p^{n-1}(K) \leqq(m-1)+s$. Furthermore the above argument can be used to show that no component of $K$ is degenerate. 
By Theorem 2.13 on p. 294 of [2], $S-K$ is $(n-1)$-ulc. This together with the hypothesis that $A$ and $B$ are each $r$-ulc, $r=1, \cdots$, $n-2$, tells us that $S-K$ is $r$-ulc, $r=1, \cdots, n-1$. (This assumes $n>1$; but the theorem is trivial if $n=1$, for then $K$ would be a finite number of points from what we have already proved.) The remainder of the proof follows exactly as the last two paragraphs of Wilder's proof of the corresponding theorem where $p^{n-1}(S)$ is assumed to be 0 (pp. 295-296 of [2]).

COROLlaRY 6.1. If $S$ is a perfectly normal connected, orientable n-gcm and $K$ is an (n-1)-dimensional closed subset of $S$, such that $S-K$ $=A \cup B$ separate, where $A$ and $B$ are $r$-ulc, $r=0,1, \cdots, n-2$, and $K$ is the common boundary of $A$ and $B$, then $A \cup K$ and $B \cup K$ are each orientable $n$-gms with boundary $K$.

Proof. By Theorem $6, K$ is an orientable $(n-1)$-gm and the conclusion follows from Theorem 1.

We now consider hypotheses under which the closure of an open subset of an orientable $n$-gcm $S$ is an orientable $n$-gm with boundary. These theorems correspond to Wilder's theorems on the boundary of uniformly locally connected subsets of a manifold. Again most of Wilder's theorems include the hypothesis $p^{n-1}(S)=0$. We shall show that these theorems can also be generalized to the case $p^{n-1}(S) \neq 0$ if certain other changes are made in the hypotheses, where as before the connectedness of the boundary is not concluded.

THEOREM 7. If $S$ is a perfectly normal connected orientable $n-\mathrm{gcm}$ and $A \subset S$ is an open ulc ${ }^{n-1}$ subset, whose boundary $K$ is $(n-1)$-dimensional, then $\bar{A}$ is an orientable $(n-1)$-gcm with boundary $K$.

Proof. By Theorem 5.19 on p. 303 of $[2], B=S-\bar{A}$ is also ulc ${ }^{n-1}$ and by Lemma 6.3 on p. 305 of [2] $K$ is also the boundary of $B$. The conclusion now follows from Corollary 6.1.

THEOREM 8. If $S$ is a perfectly normal connected orientable n-gcm and $A \subset S$ is an open ulc ${ }^{n-2}$ subset, whose boundary $K$ is $(n-1)$-dimensional and has a finite number of components, then $\bar{A}$ is an orientable $n$-gm whose boundary $K^{\prime}$ is an $(n-1)$-sub-gcm of $K$.

Proof. By Theorem 2.13 on p. 294 of [2], $S-K$ is $(n-1)$-ulc; hence $A$ and $B=S-\bar{A}$ are also both $(n-1)$-ulc. Using this and Theorem 5.19 on p. 303 of [2], we conclude that $A$ and $B$ are ulc ${ }^{n-1}$. Let $K^{\prime} \subset K$ be the boundary of $B$, then $A^{\prime}=S-\bar{B} \subset A$ is also ulc ${ }^{n-1}$ and, by Theorem $7, \bar{A}^{\prime}=\bar{A}$ is an $n$-gm with boundary $K^{\prime}$. 


\section{Bibliography}

1. P. A. White, Some characterizations of generalized manifolds with boundaries, not yet published.

2. R. L. Wilder, Topology of manifolds, Amer. Math. Soc. Colloquium Publications, vol. 32, 1949.

University of SoUthern California

\section{THE BEHAVIOR AT ZERO OF THE CHARACTERISTIC FUNCTION OF A RANDOM VARIABLE}

\section{ROSENBLATT ${ }^{1}$}

The weak laws of probability theory dealing with the asymptotic behavior of normed sums of independent and identically distributed random variables can of ten be rephrased to relate the behavior at zero of the characteristic function of a random variable to the behavior of the distribution function of the random variable at infinity. A few simple theorems of this type are here obtained.

Let $X$ be a random variable with distribution function $F(x)$ and characteristic function $\phi(z)=\int_{-\infty}^{\infty} e^{i z x} d F(x)$. Let $a(u)$ be a strictly increasing positive function defined for $u>0$ that diverges to $\infty$ as $u \rightarrow \infty . a^{-1}(u)$ will denote the inverse function of $a(u)$. The following theorems will be proved:

THEOREM 1. If

$$
\frac{a(u+1)}{a(u)}<K \quad \text { for } u \geqq 1
$$

and

$$
\frac{n}{a(n)^{2}} \int_{1}^{a(n)} \frac{x d x}{a^{-1}(x)} \leqq K, \quad \frac{n}{a(n)^{2}}=o(1) \quad \text { as } n \rightarrow \infty
$$

for some constant $K$, then

$$
1-\phi(z)=o\left(\frac{1}{a^{-1}(1 / z)}\right) \quad \text { at } z=0
$$

if and only if

Received by the editors March 2, 1951 and, in revised form, November 10, 1951.

1 This work was supported by the Office of Naval Research. 